\title{
Image Segmentation and Preserve the Boundary of the Image using B-Spline Basis
}

\author{
Gajalakshmi ${ }^{1}$ and Karunanithi $\mathrm{S}^{2}$
}

Received: 2021/ Accepted: 2021/ Published online: 21 December 2021

CSacred Heart Research Publications 2017

\begin{abstract}
This paper focuses the knot insertion in the B-spline collocation matrix, with nonnegative determinants in all $n \times n$ sub-matrices. Further by relating the number of zeros in B-spline basis as well as changes (sign changes) in the sequence of its B-spline coefficients. From this relation, we obtained an accurate characterization when interpolation by B-splines correlates with the changes leads uniqueness and this ensures the optimal solution. Simultaneously we computed the knot insertion matrix and B-spline collocation matrix and its sub-matrices having nonnegative determinants. The totality of the knot insertion matrix and B-spline collocation matrix is demonstrated in the concluding section by using the input image and shows that these concepts are fit to apply and reduce the errors through mean square error and PSNR values.
\end{abstract}

Key Words: B-spline, knots, collocation matrices, difference operator, image processing.

AMS Classification: 34B05, 34B07.

\section{Introduction}

Numerous examples are closely associated between the spline function and its B-spline function's coefficients. This is most visible in the features of the Schoenberg operator [4]. But while applying the standard well known property of matrices with dominant main diagonal, the stability of the B-spline basis, and the convergence of the control polygon

\footnotetext{
${ }^{1}$ PG \& Research Department of Mathematics, Government Thirumagal Mills College, Tamil Nadu, India, Email: n.gajalakshmi@rocketmail.com

${ }^{2}$ PG \& Research Department of Mathematics, Government Thirumagal Mills College, Tamil Nadu, India, Email: kap232008@gmail.com
} 
(B-spline) are fit to apply and reduces the errors [3, 14]. In the recent days the researchers are able to determine when interpolation using splines and B-splines are uniquely solvable based on the attribute based knots. In general, the cubic splines determine the values of the gradient based on the node's location, function value, intervals and derivatives [15]. Using local coordinates, each node and transformation technique, the period of continuity will be identified [10]. Using the first and second derivative we can estimate the input function and divide the input function / image in to $n$ equal parts. Similarly these B-splines are used to approximate the mid value of the intervals [7].

In the truncation error analysis, amplitude error and phase error analysis were produce the accurate periodic function values and these values are consistent when $h \rightarrow 0$ [8]. It is noted that the first and second derivatives of B-splines are continuous at the interior points. B-spline curve function generates a curve through control points - knots. These knots manipulate many short segments of the input function or input image $[11,13]$.

This paper is structured as follows. Section II deals with the B-spline basis through divided difference operator. The ways to construct the optimal basis of B-splines are explained in section III. Analytical expressions of the best B-spline basis are expressed in section IV. The totality of the knot insertion matrix and B-spline collocation matrix is demonstrated in the concluding section $\mathrm{V}$.

\section{B-spline basis through divided difference operator}

This section deals with B-spline basis through difference operator in an analytical manner. Consider the following sequence of non coincident, knots and $n$ be a number of curve segments of the input image [6]

$$
\mathcal{X}=\left\{x_{1}, x_{2}, \ldots, x_{n}\right\} \text { with } x_{1}<x_{2}<\cdots<x_{n}
$$

Let 


$$
D\left(\begin{array}{cccc}
u_{1}(x) & u_{2}(x) & \ldots & u_{n}(x) \\
x_{1} & x_{2} & \ldots & x_{n}
\end{array}\right)=\left|\begin{array}{cccc}
u_{1}\left(x_{1}\right) & u_{2}\left(x_{1}\right) & \ldots & u_{n}\left(x_{1}\right) \\
u_{1}\left(x_{2}\right) & u_{2}\left(x_{2}\right) & \ldots & u_{n}\left(x_{2}\right) \\
\ldots & \ldots & \ldots & \ldots \\
u_{1}\left(x_{n}\right) & u_{2}\left(x_{n}\right) & \ldots & u_{n}\left(x_{n}\right)
\end{array}\right|
$$

be the determinant of the B-spline collocation matrix which is used to preserve the boundary of the input image. Consider the knot sequence $\mathcal{U}(x)=\left\{u_{1}(x), u_{2}(x), \ldots, u_{n}(x)\right\}$ which is used to decompose the image as $n$ equal parts or $n \times n$ sub matrices. The combination of decomposed image and the normal input are together generalized as follows [16]:

$$
M\left(\begin{array}{cccc}
u_{1}(x) & u_{2}(x), & \ldots & u_{n}(x) \\
x_{1} & x_{2} & \ldots & x_{n}
\end{array}\right)=\left[\begin{array}{cccc}
u_{1}\left(x_{1}\right) & u_{2}\left(x_{1}\right) & \ldots & u_{n}\left(x_{1}\right) \\
u_{1}\left(x_{2}\right) & u_{2}\left(x_{2}\right) & \ldots & u_{n}\left(x_{2}\right) \\
\ldots & \ldots & \ldots & \ldots \\
u_{1}\left(x_{n}\right) & u_{2}\left(x_{n}\right) & \ldots & u_{n}\left(x_{n}\right)
\end{array}\right]
$$

where $D$ be the determinant and $M$ be the multiplicities of the coincident knots. Let the multiplicities of the coincident knots be defined as [2],

$$
\mathcal{M}=\left\{m_{1}, m_{2}, \ldots, m_{r}\right\} \text { with } \quad \sum_{k=1}^{r} m_{k}=n
$$

Now define

$$
\tilde{x}=\left\{x_{1}, x_{2}, \ldots, x_{n}\right\}=\{\underbrace{\eta_{1} \ldots \eta_{1}}_{m_{1}}, \underbrace{\eta_{2} \ldots \eta_{2}}_{m_{2}}, \ldots, \underbrace{\eta_{r} \ldots \eta_{r}}_{m_{r}}\}
$$

where $\eta_{1}<\eta_{2}<\cdots<\eta_{r}$ are the non coincident knots of the sequence $\tilde{x}$. Let $\mathcal{U}(x)$ are sufficiently smooth [1], and the collocation matrix associated with $\mathcal{U}(x)$ over the knot sequence (5) is given by

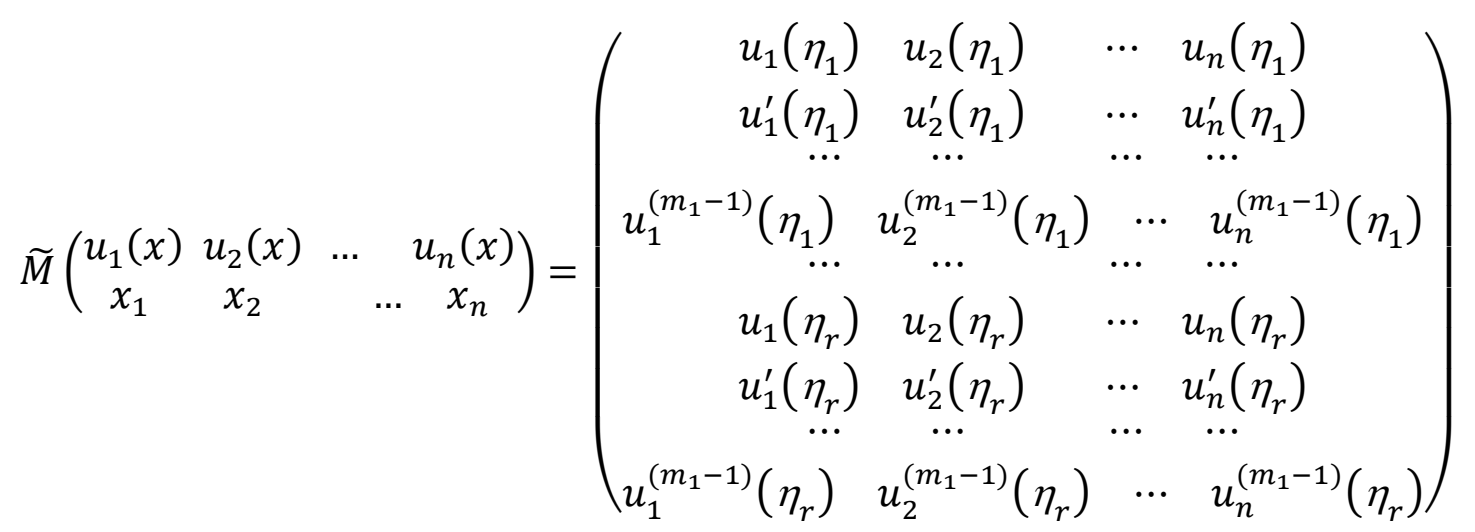


Therefore, if the performance of the above matrix has convenient derivatives, the divided difference operator will be applied in the sequence of multiple nodes $\tilde{X}$ which is often described using the collocation matrix (6).

\section{Optimal basis of B-Splines}

The optimal B-spline in bounded intervals is often described by introducing additional nodes and knots at the boundaries. Let $L \geq n+1$ be an integer and let $\mathcal{T}=\{0,1, \ldots, L-$ $1, L\}$ be the sequence of the integer knots. Extend $\mathcal{T}$ by adding $2 n$ in the $n^{\text {th }}$ element and denote it as $\tilde{\mathcal{T}}$ and is defined as

$$
\tilde{\mathcal{T}}=\{0,1, \ldots, L-1, L+2 n\}=\left\{x_{0}, x_{1}, x_{2}, \ldots, x_{2 n+L}\right\}
$$

Assume $L+2 n$ knots has the multiplicity of finite boundaries in the following interval, i.e.,

$$
\tilde{\mathcal{T}}=\left\{x_{0}, x_{1}, x_{2}, \ldots, x_{L+2 n}\right\}=\left\{\begin{array}{c}
x_{0}=x_{1}=x_{2}=\cdots,=x_{n}=0 \\
x_{k}=k-n, n+1 \leq k \leq L+n-1 \\
x_{L+n}=x_{L+n+1}=x_{L+n+2}=\cdots,=x_{L+2 n}=L
\end{array}\right.
$$

Corresponding optimal B-spline basis [5] is defined as

$$
\mathcal{N}_{n}=N_{k n}(x), 0 \leq k \leq L+2 n
$$

$N_{k n}(x)$ is having simple knots, and $L+2 n$ edge functions, is having multiple knot at the boundary. Hence the optimal B-spline basis $\mathcal{N}_{n}$ is symmetric and of degree $n$ with knots $\tilde{\mathcal{T}}$. Now compute $N_{k n}(x)$ using the following divided differences [9]

$$
N_{k n}(x)=x_{L+2 n}-x_{L}
$$

From (9), we have $\mathcal{N}_{n}$ has $L+2 n$ interior functions, i.e., $N_{k n}, 0 \leq k \leq L+2 n$ have simple

knots and $2 n$ edge functions $N_{k n}$ for $0 \leq k \leq n+\frac{L}{2}$ have multiple knots in both the boundary (during the decomposition of the input image). The basis $N_{k n}(x)$ is centrally symmetric, i.e.,

$$
N_{k n}(x)=N_{(L+n-(k+1)) n}(L-x), \quad 0 \leq k \leq n+\frac{L}{2}
$$


Consequently, the adequate basis of B-splines is stable, so that numerical errors do not seems to be magnified. The basis $N_{k n}$ is speculated and map this with the following function

$$
\mathcal{N}_{n h}=\left\{N_{k n h}(x), 0 \leq k \leq L-1+n\right\}
$$

where $L=(b-a) / h$. In general the internal functions are then calculated as follows [12]:

$$
N_{k n h}(x)=B_{n}\left(\frac{x-a}{h}-(k-n)\right), \quad n \leq k \leq L-1
$$

$2 n$ edge functions are classified as

$$
\begin{aligned}
& N_{k n h}(x)=N_{k n}\left(\frac{x-a}{h}\right), \\
& N_{(L-1+n) n h}(x)=N_{k n h}(b-x) \quad 0 \leq k \leq n-1
\end{aligned}
$$

All higher properties better construct the $\mathcal{N}_{n}$ premise once it is used in problems.

\section{B-Spline basis - Analytical expression}

Let $N_{k n}$ be the optimum basis linked with sequence of knots (7). The interior functions $N_{k n}, n \leq k \leq L-1$, are defined through B-spline $B_{n}$ as

$$
N_{k n}(x)=B_{n}(x+2 k), \quad 0 \leq k \leq L-1 \quad \text { to } 0 \leq k \leq L+1
$$

Here $N_{k n}$, having support on $0 \leq k \leq n-1$, and the right edge functions of $N_{k n}, 0 \leq k \leq$ $L+1$, having support on $0 \leq k \leq n+1$.

Theorem 1: Let $\tilde{\mathcal{T}}$ is the sequence of multiple knots in the interval I $=[0, L]$. The analytical expression of the left edge functions are given by

$$
N_{k n}(x)=\left|T_{k n}^{r}\right|(k+1) \frac{\left|T_{k n}\right|}{\left|P_{k n}\right|}, \quad 0 \leq k \leq n-1
$$

where $\left|T_{k n}\right|$ and $\left|P_{k n}\right|$ are the determinants of the following matrices

$$
T_{k n}=\left(\begin{array}{ccccc}
y^{n-k+1} & y^{n-k+2} & \cdots & y^{n} & (y-x)^{n} \\
1 & 2 & \cdots & k & k+1
\end{array}\right)
$$

$y^{n-k+1}$ to $(y-x)^{n}$ represents the number of curve segments of the input image 
$T_{k n}$ and $P_{k n}$ are the $(n+1)$ order collocation matrix

$$
P_{k n}=\left(\begin{array}{ccccc}
y^{2 n-k+1} & y 2^{n-k+2}, & \cdots & y^{2 n} & (y-x)^{2 n} \\
1 & 2 & \cdots & k & k+1
\end{array}\right)
$$

and

$$
T_{k n}^{r}=\left(\begin{array}{ccccc}
1 & 1 & \cdots & & 1 \\
2^{n-k+1} & 2^{n-k+2} & \cdots & & 2^{n} \\
\cdots & \cdots & \cdots & & \cdots \\
(r-1)^{n-k+1} & (r-1)^{n-k+2} & \cdots & (r-1)^{n} \\
(r+1)^{n-k+1} & (r+1)^{n-k+2} & \cdots & (r+1)^{n} \\
\cdots & \cdots & \cdots & & \cdots \\
(k+1)^{n-k+1} & (k+1)^{n-k+2} & \cdots & (k+1)^{n}
\end{array}\right)
$$

By expanding the determinant of $T_{k n}, P_{k n}$ as follows

Here $T_{k n}$ is the $(n-k+1)$ order diagonal matrix and constructed like

$$
T_{k n}=\operatorname{diag}(1,1,2 !, \ldots,(n-k+1))
$$

$P_{k n}$ is the $(k+1) \times(n-k+1)$ dimension matrix and constructed like

$$
P_{k n}=\left(i^{j-1}, \quad 1 \leq i \leq k+1, \quad 1 \leq j \leq n-k+1\right)
$$

and $T_{k n}^{r}$ is the $(k+1)^{t h}$ order of collocation matrix.

The statement continues to expand the determinant of $T_{k n}$ matrix. Therefore, in order to value the derivatives of the arc functions, to compute the truncated error. The left edge functions $N_{k n}, 0 \leq k \leq L-1$, holding the symmetrical property.

Corollary 1: Let $\tilde{\mathcal{T}}$ be the sequence of multiple knots in the interval $I=[0, L]$. For $0 \leq k \leq$ 2 , the left edge functions $N_{k n}$ are given by initially like

$$
\begin{gathered}
N_{0 n}(x)= \begin{cases}(1-x)^{n}, & 0 \leq x<1 \\
0, & \text { otherwise }\end{cases} \\
N_{1 n}(x)=\left\{\begin{array}{lr}
\frac{1}{2^{n}}(2-x)^{n}-2(1-x)^{n}, & 0 \leq x \leq 1 \\
\frac{1}{2^{n}}(2-x)^{n} & 1<x \leq 2, n \geq 2 \\
0, & \text { otherwise }
\end{array}\right.
\end{gathered}
$$




$$
N_{2 n}(x)=\left\{\begin{array}{lr}
\frac{1}{2} \frac{1}{3^{n-2}}(3-x)^{n}-\frac{3}{2^{n-1}}(2-x)^{n}-\frac{3}{2}(1-x)^{n} & 0 \leq x \leq 1 \\
\frac{1}{2} \frac{1}{3^{n-2}}(3-x)^{n}-\frac{3}{2^{n-1}}(2-x)^{n} & 1<x \leq 2 \\
\frac{1}{2} \frac{1}{3^{n-2}}(3-x)^{n} & 2<x \leq 3 \\
0 & \text { and } n \geq 2
\end{array}\right.
$$

It is interesting to observe that when $n=1$ (linear case), the left edge function $N_{01}(x)=$ $1-x$ and remind the basis $\mathcal{N}_{1}$ is the only one of its B-spline basis. We observed to pay particular attention to the analysis of the inputs of $x$, as it involves the values $N_{0 n}(x)$ obviously it's a positioning points. In the similar way boundary functions in the basis of cardinal B-splines localization points could be identified. Marginal B-splines are multivariable B-spline functions that are created by uni-variable B-spline basis, and we define multi-variable B-spline function in terms of matrix and an algebraic formula. The higher order derivatives of functions are extended in the form of Euler's gamma function and define like

$$
\Gamma(\beta)=\int_{0}^{\infty} z^{\beta-1} e^{-z} d z
$$

If $\Gamma(\beta)$ fulfils homogeneous initial conditions, during the interval $0 \leq k \leq m-1$, we get the following function

$$
\Gamma(\beta)=\frac{\Delta^{n+1} x^{k-\beta}}{k-\beta+1}, \quad x \geq 0, \quad 0<\beta<n
$$

where $\Delta^{n}$ is the finite difference operator. $B_{n}$ satisfies the same boundary conditions.

Finally we have

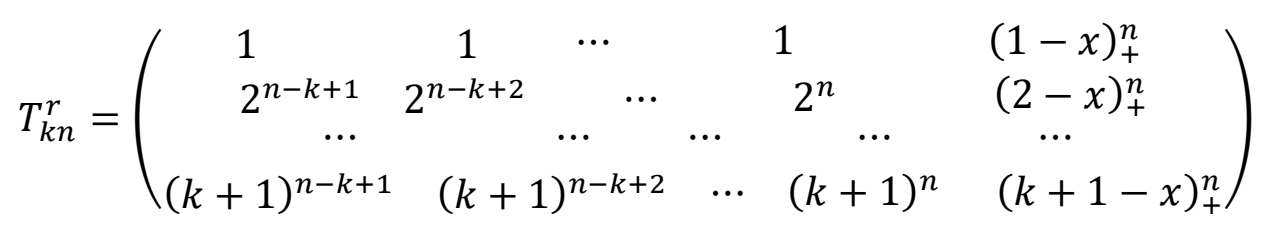




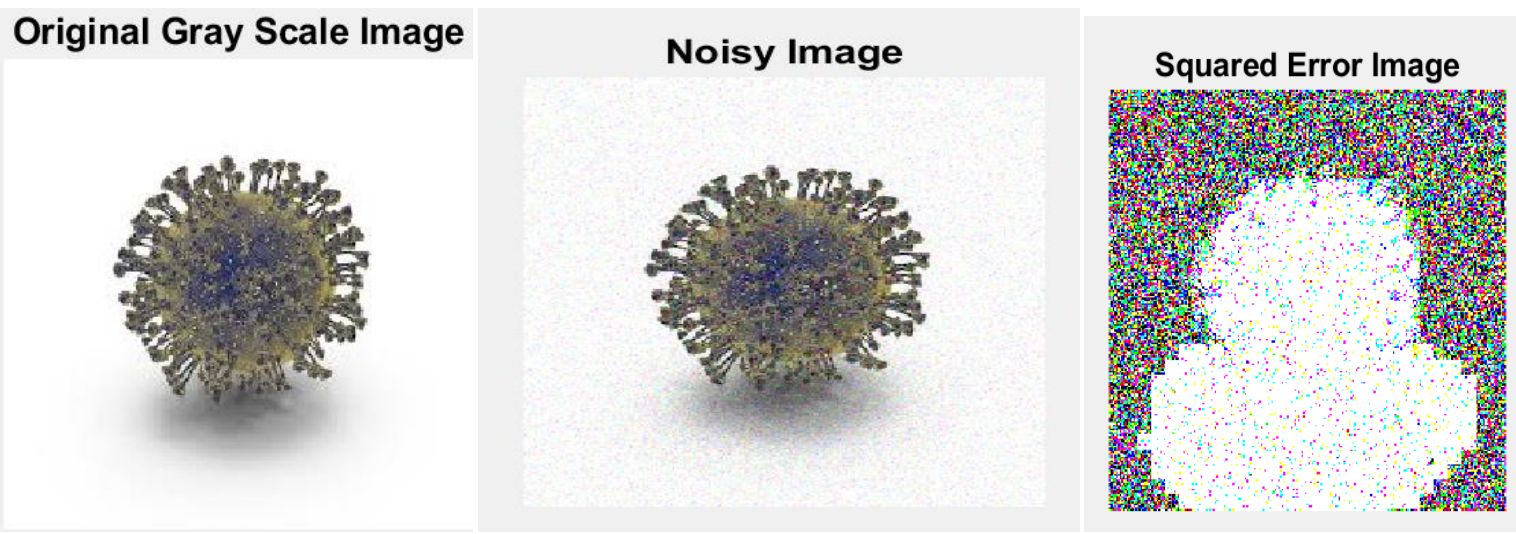

Figure 1: Input Image 1 - Original image, Noisy image and squared Error image
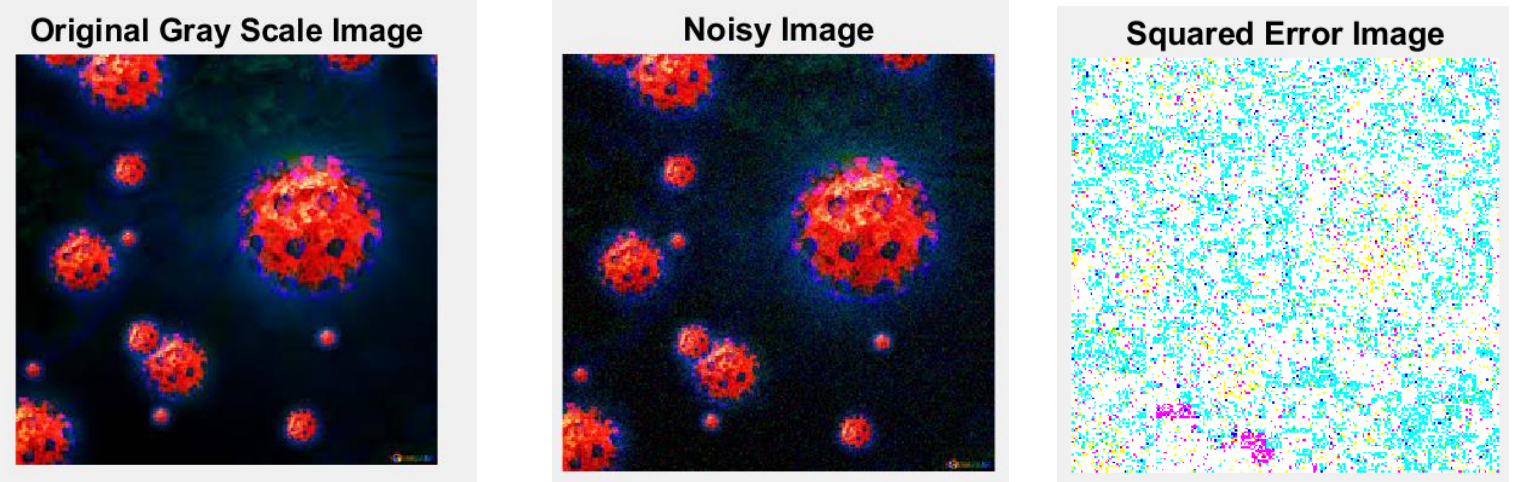

Figure 2: Input Image 2 - Original image, Noisy image and squared Error image
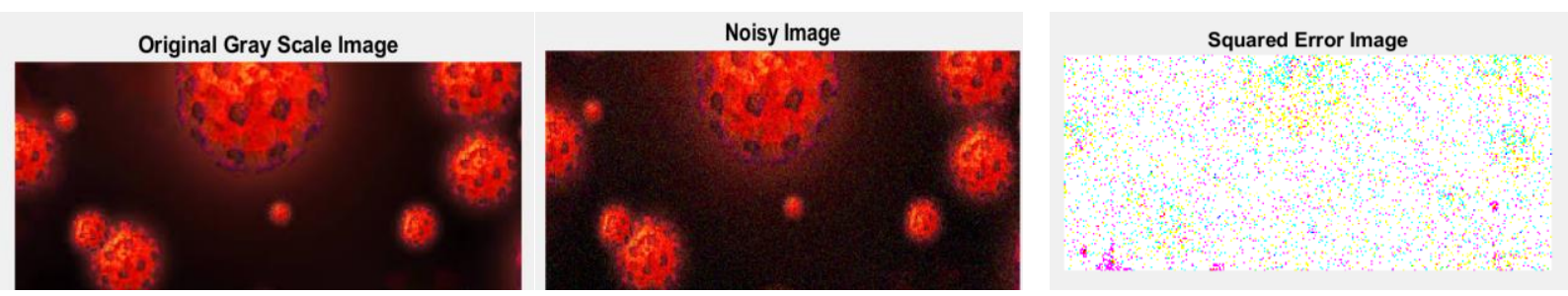

Figure 3: Input Image 3 - Original image, Noisy image and squared Error image
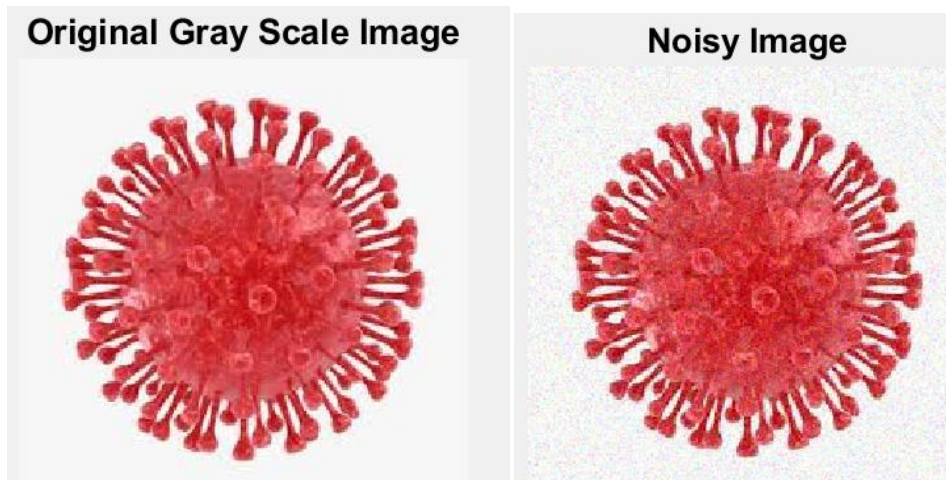

\section{Squared Error Image}


Figure 4: Input Image 4- Original image, Noisy image and squared Error image
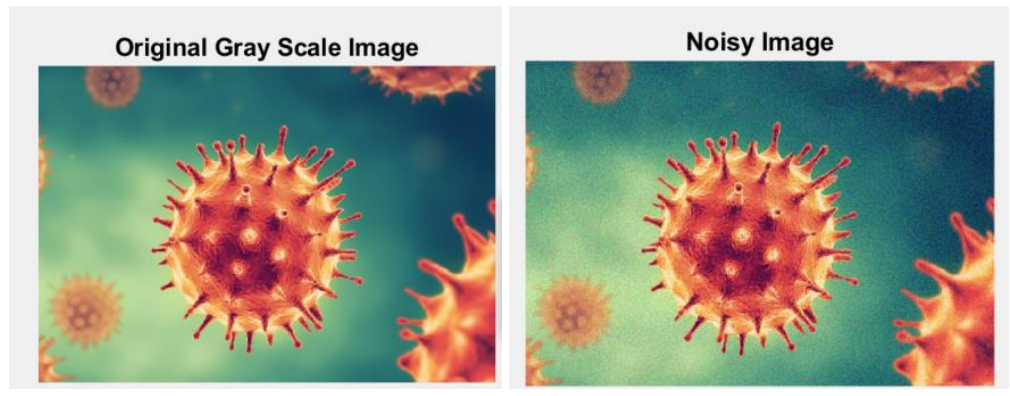

Squared Error Image

Figure 5: Input Image 5 - Original image, Noisy image and squared Error image

\begin{tabular}{|l|l|l|}
\hline Input Image & Mean Square Error & PSNR \\
\hline Input Image 1 & 42.48 & 31.93 \\
\hline Input Image 2 & 37.58 & 30.57 \\
\hline Input Image 3 & 61.08 & 31.57 \\
\hline Input Image 4 & 48.40 & 31.01 \\
\hline Input Image 5 & 61.67 & 30.04 \\
\hline
\end{tabular}

Table 1: Comparison of Mean square error and PSNR values of input images

Figures 1 to 5 represents various input images and its corresponding noisy and squared error image. Table shows the mean square error and PSNR values of the input images.

\section{Conclusion}

This study set out the digital image noise reduction through numerical analysis. Visual comparison and quantization of reconstruction of digital images from multiple images based on the basis of B-splines are computed. By fixing the computational complexity the optimal control parameters of B-spline identified in the form of matrix as well as an algebraic formula. B-spline basis is embedded with Euler's gamma function and this makes the Bspline modeling from the digital images. It is identified that that the error terms (noise) were decreased, and this leads to higher accuracy as the step size increased. By performing the 
error analysis it was concluded that the B-spline basis method is effective to reduce the noise terms of the digital images.

\section{References}

[1] Bakin S, Hegland M, Osborne M (1997), "Computational techniques and applications conference: Can mars be improved with B-splines," In Computational Techniques and Applications Conference, Adelaide, Australia.

[2] Boehm W (1980). "Inserting new knots into B-spline curves." Computer-Aided Design, 12(4), 199-201.

[3] Eilers P H, Marx B D (2010), "Splines, knots, and penalties.” Wiley Interdisciplinary Reviews: Computational Statistics, 2(6), 637-653.

[4] N Gajalakshmi and S Karunanithi, (2021), Role of recursive cubic spline interpolation method and convolution filterin image processing, J. Phys.: Conf. Ser.1964 022027.

[5] Khalid, A. Ghaffar, A, Naeem, M.N., Nisar, K.S., Baleanu, D, Solutions of BVPs arising in hydrodynamic and magnetohydro-dynamic stability theory using polynomial and non-polynomial splines.Alex. Eng. J.2021,60, 941-953.

[6] Latif, B.; Abdul Karim, S.A.;Hashim, I, (2021), New Cubic B-Spline Approximation for Solving LinearTwo-Point Boundary-Value Problems. Mathematics 9, 1250. https://doi.org/10.3390/math911125

[7] Majeed, Abdul, Muhammad Abbas, Faiza Qayyum, Kenjiro T. Miura, Md Y. Misro, and Tahir Nazir. 2020. "Geometric Modeling Using New Cubic Trigonometric BSpline Functions with Shape Parameter" Mathematics 8, No. 12: 2102. https://doi.org/10.3390/math8122102

[8] Miyata S, Shen X (2003), “Adaptive free-knot splines.” Journal of Computational and Graphical Statistics, 12(1), 197-213.

[9] Munguia, M.; Bhatta, D, (2015), Use of Cubic B-Spline in Approximating Solutions of Boundary Value Problems.Appl. Appl. Math. Int. J.,10, 750-771.

[10] Nazir T, Abbas M, Iqbal M.K, (2020), New cubic B-spline approximation technique for numerical solutions of coupled viscous Burgers equations. Eng. Comput. Swans. Wales, 38, 83-106.

[11] Peng Weilong, Su Yong, Tang Keke, Xu Chao, Feng Zhiyong, Fang Meie (2021), Geometrical Consistency, Modeling on B-Spline Parameter Domain for 3D Face Reconstruction From Limited Number of Wild Images, Frontiers in Neurorobotics 
Vol 15, https://www.frontiersin.org/article/10.3389/fnbot.2021.652562, ISSN 1662 5218, DOI:10.3389/fnbot.2021.652562.

[12] Richardson S, Wang S and Jennings LS (2008), "A multivariate adaptive regression B-spline algorithm (BMARS) for solving a class of nonlinear optimal feedback control problems." Automatica, 44(4), 1149-1155.

[13] Tassaddiq, A.; Khalid, A.; Naeem, M.N.; Ghaffar, A.; Khan, F.; Karim, S.A.A.; Nisar, K.S. (2019), A new scheme using cubic B-spline tosolve non-linear differential equations arising in visco-elastic flows and hydrodynamic stability problems.Mathematics, 7, 1078.

[14] M. Unser, A. Aldroubi, and M. Eden, "Fast B-splines transforms for continuous image representation and interpolation," IEEE Trans. Pattern Anal. Machine Intell., vol. 13, pp. 277-285, 1991.

[15] Zhou S, Shen X (2001), "Spatially adaptive regression splines and accurate knot selection schemes.” Journal of the American Statistical Association, 96(453), 247-259.

[16] Y. Yuan, N. Chen, and S. Zhou. Adaptive b-spline knot selection using multiresolution basis set. IIE Transactions, 45(12):1263-1277, 2013. 